\title{
APPLICATION OF SUPPORT VECTOR MACHINES CLASSIFIERS TO VISUAL SPEECH RECOGNITION
}

\author{
Mihaela Gordan*, Constantine Kotropoulos ${ }^{* *}, \quad$ Ioannis Pitas ${ }^{* *}$ \\ * Technical University of Cluj-Napoca \\ Cluj-Napoca, Romania \\ mihag@bel.utcluj.ro \\ ** Department of Informatics \\ Aristotle University of Thessaloniki \\ Box 451, Thessaloniki 540 06, GREECE \\ \{costas, pitas\}@zeus.csd.auth.gr
}

\begin{abstract}
In this paper we proposed a visual speech recognition network based on Support Vector Machines. Each word of the dictionary is modeled by a set of temporal sequences of visemes. Each viseme is described by a support vector machine, and the temporal character of speech is modeled by integrating the support vector machines as nodes into Viterbi decoding lattices. Experiments conducted on a small visual speech recognition task show a word recognition rate on the level of the best rates previously reported, even without training the state transition probabilities in the Viterbi lattices and using very simple features. This proves the suitability of support vector machines for visual speech recognition.
\end{abstract}

\section{INTRODUCTION}

The recognition of speech from the visual information only is referred as visual speech recognition or lipreading. Different shapes of the mouth (i.e. different mouth openings, different position of the teeth and tongue) realized during speech cause the production of different phones. A mouth shape and mouth dynamics corresponding to the production of a phone or a group of phones indistinguishable in the visual domain defines a viseme [6]. One can establish a correspondence between visemes and phonemes, even if this correspondence is not one-to-one, but one-to-many, due to the involvement of non-visible parts of the vocal tract in the speech production. Still, for word dictionary of small size, we can perform good quality speech recognition using only a viseme-level description of the words.

Many methods have been proposed for solving the visual speech recognition problem in the literature. The different types of solutions adopted vary widely with respect to: the feature types; the classifier used; the class definition. For example, Bregler uses time-delayed neural networks (TDNN) for visual classification, and the outer lip contour coordinates as visual features [4]. Luettin uses active shape models for representing different mouth shapes, gray level distribution profiles (GLDPs) around the outer and/or inner lip contours as feature vectors, and finally builds whole-word hidden Markov models (HMM) for visual speech recognition [5].

This work was supported by the European Union Research Training Network "Multi-modal Human-Computer Interaction (HPRN-CT-200000111).
Movellan employs also HMMs for building visual word models, but uses directly the gray levels of mouth images as features after some simple preprocessing to exploit the vertical symmetry of the mouth [3].

Despite the variety of existing strategies for visual speech recognition, there is still ongoing research in this area, attempting to: 1) find the most suitable features and classification techniques to discriminate as good as possible between different mouth shapes, but to keep in the same class the mouth shapes corresponding to the same phone produced by different individuals (i.e., to be individual-independent) thus leading to higher visual speech recognition rates; 2) require as few processing of the mouth image as possible, to allow the implementation in real time of the mouth shape classifier considering that the end use of mouth shape classifier is in audio-visual speech recognition systems, which are supposed to work in real-time; 3) facilitate the easy integration of audio and video speech recognition.

In this paper, we aim to contribute to the first two aspects mentioned above by examining the suitability of support vector machines (SVMs) for visual speech recognition tasks motivated by the fact that SVMs have been proved powerful classifiers in various pattern recognition applications such as face detection, face recognition, etc., to mention a few. Very good results in audio speech recognition using SVMs were recently reported in [1]. No attempts in applying SVMs for visual speech recognition have been reported so far, although a somehow closely related application is described in [2], where SVMs were applied for detecting the degree of opening/smile of mouth images in videosequences. This work uses SVMs for linear regression, not for classification task. Thus, according to the best of the author's knowledge, the use of SVMs as visual speech classifiers is a novel idea.

One of the reasons for not using SVMs in audiovisual speech recognition so far is the fact that they are inherently static classifiers, whilst speech is a dynamic process, where the temporal information is essential for recognition. A solution to mitigate this deficiency is presented in [1], where a combination of HMM with SVM is proposed. In this paper we adopt a similar strategy for modeling the visual speech dynamics with the difference that we shall use only the Viterbi algorithm employed by an HMM to create dynamically visual word models. Another novel aspect in the visual speech recognition approach proposed here refers to 
the strategy adopted for building the word models: while most of the applications presented in the literature $[1,5,3]$ build whole word models as basic visual models, our basic visual models are viseme-oriented models, and the visual word model is obtained by the combination of these basic models into a temporal dynamic sequence. This approach offers the advantage of an easier generalization to larger vocabulary word recognition tasks without significantly increasing the storage requirements by maintaining the dictionary of basic visual models needed for word modeling into a reasonable limit. Although using this viseme-oriented word modeling approach we could expect some performance hit in the word recognition rate, the experimental results are on the level of the best previous reported in literature even without learning the state transition probabilities, which is very encouraging. In the case of using very simple features (i.e. pixels), our word recognition rate is superior to the ones reported in the literature. The viseme-oriented approach can also facilitate the integration of audio and visual speech recognition when phoneme-based audio speech recognition is employed.

\section{OVERVIEW OF SUPPORT VECTOR MACHINES}

SVMs is a principled technique to train classifiers that stems from statistical learning theory $[7,8]$. Their root is the optimal hyperplane algorithm. They minimize a bound on the empirical error and the complexity of the classifier at the same time. Accordingly, they are capable of learning in sparse high-dimensional spaces with relatively few training examples. Let $\left\{\mathbf{x}_{i}, y_{i}\right\}, i=$ $1,2, \ldots, N$, denote $N$ training examples where $\mathbf{x}_{i}$ comprises an $M$-dimensional pattern and $y_{i}$ is its class label. Without any loss of generality we shall confine ourselves to the two-class pattern recognition problem. That is, $y_{i} \in\{-1,+1\}$. We agree that $y_{i}=+1$ is assigned to positive examples, whereas $y_{i}=-1$ is assigned to counterexamples.

The data to be classified by the SVM might be linearly separable in their original domain or not. If they are separable, then a simple linear SVM can be used for their classification. However, the power of SVMs is demonstrated better in the nonseparable case, when the data cannot be separated by a hyperplane in their original domain. In the latter case, we can project the data into a higher dimensional Hilbert space and attempt to linearly separate them in the higher dimensional space using kernel functions. Let $\Phi$ denote a nonlinear map $\Phi: \mathcal{R}^{M} \rightarrow \mathcal{H}$ where $\mathcal{H}$ is a higher-dimensional Hilbert space. SVMs construct the optimal separating hyperplane in $\mathcal{H}$. Therefore, their decision boundary is of the form:

$$
f(\mathbf{x})=\operatorname{sign}\left(\sum_{i=1}^{N} \alpha_{i} y_{i} K\left(\mathbf{x}, \mathbf{x}_{i}\right)+b\right)
$$

where $K\left(\mathbf{z}_{1}, \mathbf{z}_{2}\right)$ is a kernel function that defines the dot product between $\Phi\left(\mathbf{z}_{1}\right)$ and $\Phi\left(\mathbf{z}_{2}\right)$ in $\mathcal{H}$, and $\alpha_{i}$ are the nonnegative Lagrange multipliers associated with the quadratic optimization problem that aims to maximize the distance between the two classes measured in $\mathcal{H}$ subject to the constraints

$$
\begin{array}{ll}
\mathbf{w}^{T} \Phi\left(\mathbf{x}_{i}\right)+b \geq 1 & \text { for } y_{i}=+1 \\
\mathbf{w}^{T} \Phi\left(\mathbf{x}_{i}\right)+b \leq 1 & \text { for } y_{i}=-1 .
\end{array}
$$

Frequently used kernel functions are:

1) the polynomial kernel:
$\mathbf{K}\left(\mathbf{x}_{i}, \mathbf{x}_{j}\right)=\left(m \mathbf{x}_{i}^{T} \mathbf{x}_{j}+n\right)^{d}$

2) the Radial Basis Function (RBF) kernel:

$\mathbf{K}\left(\mathbf{x}_{i}, \mathbf{x}_{j}\right)=\exp \left\{-\gamma\left|\mathbf{x}_{i}-\mathbf{x}_{j}\right|^{2}\right\}$.

In the following, we will omit the sign function from the decision boundary (1) that simply makes the optimal separating hyperplane an indicator function.

To enable the use of SVMs in visual speech recognition, when we model the speech as a temporal sequence of symbols corresponding to the different phones produced, we shall employ the SVMs as nodes in a Viterbi lattice. The nodes of such a Viterbi lattice are supposed to generate the posterior probabilities of the corresponding symbols to be emitted [10], and the standard SVMs do not provide such probabilities as output. Several solutions are proposed in the literature to map the SVM output to probabilities: the cosine decomposition proposed by Vapnik [7], the probabilistic approximation by applying the evidence framework to SVMs [11], the sigmoidal approximation by Platt [12]. Here we adopt the solution proposed by Platt [12], since it is a simple solution which was already used in a similar application of SVMs to audio speech recognition [1]. This solution shows that having a trained SVM, we can convert its output to probability by training the parameters of a sigmoidal mapping function:

$$
P(y=+1 \mid f(\mathbf{x}))=\frac{1}{1+\exp \left(a_{1} f(\mathbf{x})+a_{2}\right)}
$$

where $a_{1}$ and $a_{2}$ are the parameters of the sigmoidal mapping to be derived for the trained SVM under consideration with $a_{1}<0$. $P(y=+1 \mid f(\mathbf{x}))$ gives directly the posterior probability to be used in the Viterbi decoder. The parameters $a_{1}$ and $a_{2}$ are derived from the training set $\left\{f\left(\mathbf{x}_{i}\right), y_{i}\right\}, i=1,2, \ldots, N$, using maximum likelihood estimation. The detailed description of the training algorithm can be found in [12]. Platt shows on experimental real data that the sigmoidal model of posterior probabilities by equation (3) approximates very well the real distribution even for the case of non-Gaussian distributions, when the real-valued output function of the SVM presents discontinuities around the margins [12].

\section{THE PROPOSED APPROACH TO VISUAL SPEECH RECOGNITION}

The problem of discriminating between different mouth shapes during speech production can be viewed as a pattern recognition problem. In this case, the set of patterns is a set of feature vectors $\left\{\mathbf{x}_{i}\right\}, i=1,2, \ldots, P$, each of them describing some mouth shape. The feature vector $\mathbf{x}_{i}$ is a representation of the mouth image (either low-level, such as the gray levels from a rectangular image region containing the mouth, geometric parameters such as the mouth width, height, perimeter, or the coefficients of a linear transformation of the mouth image). All the feature vectors from the set have the same number of components, $M$.

Let us denote the pattern classes by $\mathcal{C}_{j}, j=1,2, \ldots, Q$ where $Q$ is the total number of classes. Each pattern class $\mathcal{C}_{j}$ is a group of patterns that represent mouth shapes corresponding to the same viseme. The class label of the class $\mathcal{C}_{j}$ is denoted by $l_{j}$.

A set of $Q$ parallel SVMs is built where each SVM is trained to classify test patterns in class $\mathcal{C}_{j}$ or its complement $\mathcal{C}_{j}^{C}$ (i.e., not in class $\mathcal{C}_{j}$ ). The set of $Q$ binary SVMs will ensure the multiclass classification of any test pattern to one of the $Q$ classes. We use the 1-vs-all multiclass SVM strategy [13]. To derive an unambiguous classification, we use SVMs with probabilistic outputs 
[12], namely, the output of each SVM classifier $S V M_{l}$ is the posterior probability for the test pattern $x_{k}$ to belong to the class $\mathcal{C}_{l}$, $l=1,2, \ldots, Q, P\left(y_{l}=1 \mid f_{l}\left(\mathbf{x}_{k}\right)\right)$.

This pattern recognition problem can be applied to visual speech recognition in the following way: each unknown pattern represents the image of the speaker's face at a certain time instant; each class label represents one viseme. Accordingly, we can compute the probability for each viseme to be produced at any time instant in the spoken sequence. Correlations can be established between the different phones produced during speech and the visemes corresponding to them. The solution adopted is to define the viseme classes and the viseme-to-phoneme mapping dependent on the application (i.e., the recognition of the first four digits in English, as spoken by the different individuals in the Tulips1 database [3]). The 12 viseme classes defined and their corresponding phonemes are: • for phonemes $W, U W$ and $A O$, the visemes: $w, a o, w a o$; $\bullet$ for phoneme $A H$, the viseme $a h$; $\bullet$ for phoneme $N$, the viseme $n$; - for phoneme $T$, the viseme $t$; $\bullet$ for phoneme $T H$, the visemes: $t h_{1}, t h_{2}$; • for phoneme $R$, the visemes: $w, a o$; $\bullet$ for phoneme $I Y$, the visemes: $i y, a h ; \bullet$ for phoneme $F$, the visemes: $f_{1}, f_{2}$, $f_{3}$.

By its nature, speech is a temporal process. Each spoken word can be modelled in the visual domain as a sequence of visemes corresponding to some basic sounds, called here visemic model. Having defined the viseme-to-phoneme mapping for our application and having the phonetic description of each word from the dictionary, we can build the symbolic visemic models of the words in the dictionary. For our application, we will have a set of 28 visemic models. The most natural way of representing the word models in the temporal domain, starting only from the symbolic visemic model and from the total number of $T$ frames in the word pronunciation, is to assume that the duration of each viseme in the word pronunciation can be whatever, but necessarily not zero. Thus, for each symbolic visemic model, we can create a temporal network, containing as many states as many frames we have in the videosequence, that is, $T$. The most straightforward way to represent such a temporal network is the Viterbi algorithm [10]. The resulting Viterbi lattice is shown in Figure 1 for one symbolic visemic model of the word "one". The paths formed by the solid lines show the possible model realizations. Each node signifies the realization of the corresponding viseme at that particular time instant. Each visemic model of a word from the dictionary, denoted $w_{d}, d=1,2, \ldots, D$, will have its own Viterbi lattice. Let us in-

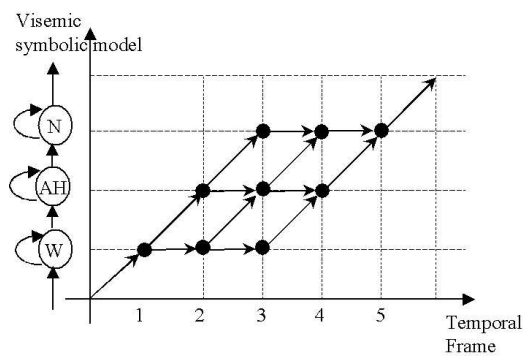

Fig. 1. The temporal Viterbi lattice for the pronunciation of the word "one" in a videosequence of 5 frames

terpret each node in the lattice of Figure 1 as the probability that the corresponding symbol $o_{k}$ is emitted at the time instant $k$. We denote this probability by $b_{o_{k} k}$. Each solid line between the nodes corresponding to the symbol $o_{k}$ at the time instant $k$ and $o_{k+1}$ at the time instant $k+1$ represents the transition probability from the state that is responsible for the generation of $o_{k}$ to the state that generates the symbol $o_{k+1}$. We denote the latter probability by $a_{o_{k} o_{k+1}}$, where $o_{k}$ and $o_{k+1}$ may be different or not.

Having a videosequence of $T$ frames for a word pronounced and such a Viterbi model for each visemic word model $w_{d}, d=$ $1,2, \ldots, D$, we can compute the probability for the visemic word model $w_{d}$ to be produced following a path $\ell$ in the Viterbi lattice as:

$$
p_{d, \ell}=\prod_{k=1}^{T} b_{o_{k} k} \cdot \prod_{k=1}^{T-1} a_{o_{k} o_{k+1} \mid d, \ell} .
$$

and the probability for the visemic word model $w_{d}$ to be produced as the maximum over all possible $p_{d, \ell} \mathrm{s}$. Among the words that can be produced following all the possible paths in all the $D$ Viterbi lattices, the most probable word, that is, the word corresponding to the model $d$ whose probability $p_{d}, d=1,2, \ldots, D$, is maximum is finally recognized. In the visual speech recognition approach discussed in this paper, the symbol emission probabilities $b_{o_{k} k}$ are given by the corresponding SVMs, $S V M_{o_{k}}$. To a first approximation, we assume equal transition probabilities $a_{o_{k} o_{k+1}}$ between whatever two symbol emission states.

The complexity of the SVM structure can be estimated by the number of SVMs needed for the classification of each word, as a function of the number of frames $T$ in the current word pronunciation. Considering the total number of symbolic word models and the number of possible states as a function of the frame index, we get: 9 SVMs needed for the classification of the first frame, 6 for the last and before-last frame, 11 for the second frame and all 12 SVMs for any other frame. This leads to a total of $12 \times T-16$ SVMs.

\section{EXPERIMENTAL RESULTS}

To evaluate the recognition performances of the proposed SVMbased visual speech recognizer, we choose to solve the task of recognizing the first four digits in English from the small audiovisual database Tulips1 [3], frequently used in similar visual speech recognition experiments. First we define the viseme classes for each word, based on their phonetic descriptions [14] trough the manual annotation of the training set, then train one SVM for each viseme considered. We used for our experiments SVMs with polynomial kernel of degree 3 . We used two types of features: 1) The first type comprises the gray levels of a rectangular region of interest around the mouth, downsampled to the size $16 \times 16$ and scanned raw by raw. Each mouth image is represented by a feature vector of length 256. 2) The second type represents each mouth image frame at the time $T_{f}$ by a vector of double size i.e. $2 \times 256=512$, that comprises the gray levels of the rectangular region of interest as previously, and the temporal derivatives of the gray levels normalized to the range $\left[0, L_{\operatorname{Max}}-1\right]$ (where $L_{\operatorname{Max}}$ is the maximum gray level value in mouth image). The temporal derivatives are simply the pixel by pixel gray level differences between the frames $T_{f}$ and $T_{f}-1$ and are called delta features.

The complete visual speech recognizer was implemented in $\mathrm{C}++$. We used the publicly available SVMLight toolkit modules for the training of the SVMs [9] and implemented in C++ the module for learning the sigmoidal mapping of the SVMs output to probabilities and the module for generating the Viterbi decoding lattices based on SVMs with probabilistic outputs. 
Table 1. The overall WRR of the SVM dynamic network compared to other techniques.

\begin{tabular}{|l|l|l|l|l|l|l|}
\hline Method & $\begin{array}{l}\text { SVM-based } \\
\text { dynamic net- } \\
\text { work without } \\
\text { delta features }\end{array}$ & $\begin{array}{l}\text { SVM-based dy- } \\
\text { namic network } \\
\text { with delta fea- } \\
\text { tures }\end{array}$ & $\begin{array}{l}\text { AAM and HMM } \\
\text { system (shape + in- } \\
\text { tensity model, inner } \\
\text { + outer lip contour) } \\
\text { no delta features [5] }\end{array}$ & $\begin{array}{l}\text { AAM and HMM } \\
\text { system (shape + in- } \\
\text { tensity model, inner } \\
\text { + outer lip contour) } \\
\text { + delta features [5] }\end{array}$ & $\begin{array}{l}\text { Stochastic net- } \\
\text { works, no delta } \\
\text { features [3] }\end{array}$ & $\begin{array}{l}\text { Stochastic net- } \\
\text { works, + delta } \\
\text { features [3] }\end{array}$ \\
\hline WRR [\%] & 76 & 87.5 & 90.6 & 60 & 89.93 \\
\hline
\end{tabular}

We performed speaker-independent visual speech recognition tests, using the leave-one-out testing strategy for the 12 subjects in the Tulips1 database. More precisely, the testing strategy was as follows: we trained the system 12 times separately, each time using 11 subjects in the training set and leaving the $12^{\text {th }}$ subject out for testing. In this way, we obtained a total of 96 video test sequences. We examine the overall percentaged word recognition rate WRR, comparing this result with the ones reported in literature under similar conditions (i.e., using the same features, the same database and the same testing procedure) $[5,3]$ in Table 1. We can see that our results are on the same level as the best ones reported in the literature $(W R R=90.6 \%)$. However the features used by us are simpler than those used in literature to obtain the same WRR. For the shape + intensity models [5] the gray levels should be sampled in the exact subregion of the mouth image containing the lips, around the inner and outer lip contours, and should exclude the skin areas. Accordingly, the method reported in [5] requires the tracking of the lip contour in each frame, which increases the processing time of visual speech recognition. Moreover we notice that our very good WRR was obtained without training the transition probabilities in the Viterbi decoding lattice from whole-word models, which could cause a performance hit. The fact that the results are as good as the ones given by whole word models is promising. An improvement of the WRR is expected when training of the transition probabilities is implemented and the trained transition probabilities are incorporated in the Viterbi decoding lattices.

\section{CONCLUSIONS}

We examined the suitability of SVMs with probabilistic outputs in visual speech recognition by employing them into a dynamic temporal network implemented by a number of Viterbi decoding lattices as nodes and testing the proposed method on a small visual speech recognition task. Using very simple techniques, we obtained good word recognition rates as compared to the state of the art results from the literature. This demonstrates that SVMs are promising classifiers for visual speech recognition tasks. Another advantage of the viseme-oriented modeling method proposed is the possibility of easier generalization to larger vocabularies. In our future research, we will try to improve the performance of the visual speech recognizer by using other kernel functions, learning the state transition probabilities of the Viterbi decoding lattices and using better strategies for multiclass SVM implementation. Also we intend to analyze the performance of our approach for larger vocabulary tasks.

\section{REFERENCES}

[1] A. Ganapathiraju, J. Hamaker, and J. Picone. "Hybrid SVM/HMM architectures for speech recognition," in Proc. of Speech Transcription Workshop, College Park, Maryland, USA, May 2000.

[2] V. P. Kumar and T. Poggio. "Learning-based approach to real time tracking and analysis of faces," in Proc. of AFGR, 2000.

[3] J. R. Movellan. "Visual speech recognition with stochastic networks," in Advances in Neural Information Processing Systems, (G. Tesauro, D. Toruetzky, and T. Leen, Eds.), Vol 7, MIT Press, Cambridge, MA, 1995

[4] C. Bregler and S. Omohundro. "Nonlinear manifold learning for visual speech recognition," in Proc. IEEE ICCV, 1995, pp. 494-499.

[5] J. Luettin and N. A. Thacker. "Speechreading using probabilistic models," Computer Vision and Image Understanding, 65(2):163-178, February 1997

[6] C. Benoît, T. Lallouache, T. Mohamadi, and C. Abry. "A set of French visemes for visual speech synthesis," in Talking machines: Theories, Models, and Designs, (G. Bailly and C. Benoît, Eds.), 485-504, North Holland, Elvsevier, Amsterdam, 1992 J. R. Movellan.

[7] V.N. Vapnik. Statistical Learning Theory, J. Wiley, N.Y., 1998

[8] N. Cristianini and J. Shawe-Taylor. An introduction to support vector machines, Cambridge University Press, Cambridge, U.K., 2000

[9] T. Joachims. "Making large-scale SVM learning practical," in Advances in Kernel Methods - Support Vector Learning, (B. Scoelkopf, C. Burges, and A. Smola, Eds.), MIT-Press, 1999

[10] S. Young, D. Kershaw, J. Odell, D. Ollason, V. Valtchev, and P. Woodland. The HTK book, Entropic, Ltd., Cambridge, UK, HTK version 2.2 edition, 1999

[11] J.T.-Y. Kwok. "Moderating the outputs of support vector machine classifiers," in IEEE Transactions on Neural Networks, 10(5):1018-1031, September 1999

[12] J. Platt. "Probabilistic outputs for support vector machines and comparisons to regularized likelihood methods," in $\mathrm{Ad}$ vances in Large Margin Classifiers, (A. Smola, P. Bartlett, B. Scholkopf, and D. Schuurmans, Eds.), MIT Press, Cambridge, MA, 2000

[13] D.J. Sebald and J.A. Bucklew. "Support vector machines and the multiple hypothesis test problem," in IEEE Transactions on Signal Processing, 49(11):2865-2872, November 2001

[14] The Carnegie Mellon University Pronouncing Dictionary v. 0.6. http://www.speech.cs.cmu.edu/cgi-bin/cmudict 